\title{
IMAGE-BASED 3D RECONSTRUCTION AND ANALYSIS FOR ORTHODONTIA
}

\author{
V. A. Knyaz*, \\ State Research Institute of Aviation Systems (GosNIIAS), 125319, 7, Victorenko str., Moscow, Russia - \\ knyaz@gosniias.ru
}

Commission V, WG V/4

KEY WORDS: Close-range Photogrammetry, Accuracy, Orthodontia, Visualization

\begin{abstract}
:
Among the main tasks of orthodontia are analysis of teeth arches and treatment planning for providing correct position for every tooth. The treatment plan is based on measurement of teeth parameters and designing perfect teeth arch curve which teeth are to create after treatment. The most common technique for teeth moving uses standard brackets which put on teeth and a wire of given shape which is clamped by these brackets for producing necessary forces to every tooth for moving it in given direction. The disadvantages of standard bracket technique are low accuracy of tooth dimensions measurements and problems with applying standard approach for wide variety of complex orthodontic cases. The image-based technique for orthodontic planning, treatment and documenting aimed at overcoming these disadvantages is proposed. The proposed approach provides performing accurate measurements of teeth parameters needed for adequate planning, designing correct teeth position and monitoring treatment process. The developed technique applies photogrammetric means for teeth arch $3 \mathrm{D}$ model generation, brackets position determination and teeth shifting analysis.
\end{abstract}

\section{INTRODUCTION}

Being aimed for transforming patient's teeth arches to a perfect condition orthodontia needs accurate data of teeth dimensions, shape, position and orientation relatively to a reference system of coordinates. This information allows determining the perfect teeth arches shape, which has to be the result of treatment. To move teeth to the perfect position special techniques are used. The most common one uses special brackets which put on teeth and a wire of given shape which is clamped by these brackets for producing necessary forces to every tooth for moving it in given direction.

Most of existing techniques use orthodontic wires of standard shape for treatment. Unfortunately it is an often case when standard wire is not suit for individual person. So there is a need for designing individual wire for every case. To solve this problem the image based system for orthodontic treatment planning and analysis is developed. It uses a plaster copy of teeth arch for 3D reconstruction and measurements of required teeth geometric parameters for orthodontic analysis of a given case and for designing individual orthodontic wire. For 3D reconstruction a photogrammetric technique is used. 3D reconstruction system includes two high resolution CCD cameras, structured light projector and rotation stage for automatic whole 3D model reconstruction. It allows generating of teeth arches 3D model in automatic mode and solving occlusion registration problem (the problem of putting virtual teeth arches in the mutual position according to a real case). For this purpose plaster models are installed in given posture using dental articulator or plaster form for setup. Then mutual position of jaws is registered by the developed photogrammetric system. Two methods of occlusion registration are developed and tested. The first method uses a set of reference points on the upper and lower teeth arches. Images of teeth arches are captured by the photogrammetric system, and then 3D coordinates of reference points are calculated for mutual jaws position determination. When applying the second method the surface of upper and lower teeth rows is scanned in given position of teeth arches plaster models. Then the teeth arches 3D model mutual position is determined using a set of this surface scans as a reference surface using iterative closest point algorithm. The estimation of method accuracy and comparative analysis of these two techniques for jaw 3D models translating were performed.

For optimal orthodontic wire designing virtual bracket is placed on virtual tooth in required position by expert. Then the developed software allows designing perfect teeth arch for given case using measured information about teeth and to design optimal wire (or set of wires) for moving teeth to perfect position.

Photogrammetric approach allows getting accurate and convenient solution for concerned problems. Application of an accurate teeth arch 3D model instead plaster mould gives to a orthodontist new possibilities for occlusion analysis, treatment planning and visualization of treatment forecast using patient's face 3D model.

Proposed photogrammetric system supports all necessary functions for teeth arches occlusion analysis and treatment planning. It uses specially designed hardware for generating a teeth arch 3D model and face 3D model in automated mode and original software for models setting up, occlusion analysis and optimal wire design.

\footnotetext{
* Corresponding author.
} 
This technique allows performing necessary orthodontic analysis of mutual teeth location. An orthodontist can define perfect teeth arches mutual position and to plan a treatment (to design a set of wires). Also this technique allows controlling the process of orthodontic treatment.

\section{EXISTING TECHNIQUES}

Orthodontia is a science which determines the perfect position and orientation of teeth and develops techniques for moving teeth to this perfect position. Unfortunately it is an often case when teeth in a teeth arch are not located perfectly. So the task of orthodontia is to set teeth in a perfect position.

Different techniques were developed to correct teeth position by applying given forces to teeth. For producing such forces wires and brackets or kappa are traditionally used. For successful treatment planning accurate data about teeth arch is needed. Traditional techniques use plaster mould of teeth arch for treatment planning. In most cases teeth dimensions needed for treatment planning are determined by a ruler and a size of working wire is chosen from a set of three or four standard types. Unfortunately it sometimes causes problems in treatment.

To overcome these problems some groups develop individual brackets and kappa which are designed for determined person. So Align Technology Inc. proposes a set of invisible kappa produced using teeth arch 3D model. Every kappa is used on a planned stage of treatment corresponding to the current position of teeth.

3M Company develops Invisible Incognito ${ }^{\mathrm{TM}}$ Orthodontic Braces. A set of lingual individual brackets and wires are offered for a staged treatment. Iindividual brackets are created using accurate 3D models of tooth surface. Such an approach provides better contact between a tooth and a bracket and therefore less probability of a bracket getting off the tooth. But in this technique manual stage exists on which plaster mould is physically divided into separated teeth (with the help of a saw) and then orthodontist places separate teeth to a perfect position manually using cement.

These techniques use 3D teeth arch models for treatment support. But a lot of hand work still is to be done by an orthodontist.

Most of existing techniques use standard wires and standard brackets for creating forces which are to move tooth in a correct position. The disadvantages of such a technique are complex procedure of accurate bracket positioning on a plaster mould of a teeth arch and then accurate translation this brackets from plaster mould to real teeth arch.

The standard procedure of setting up a standard wire includes the next stages:

- Producing plaster copy of teeth arch

- Positioning standard brackets on the plaster copy of teeth arch (Fig. 1.) using special device (articulator) which provides required bracket position on a tooth for producing correct forces for required tooth movement

- Making a silicon kappa containing installed brackets in required position
- Placing the brackets on a patient's teeth using this kappa which provide accurate brackets transfer to a given position for every tooth

- Placing a standard wire into the brackets

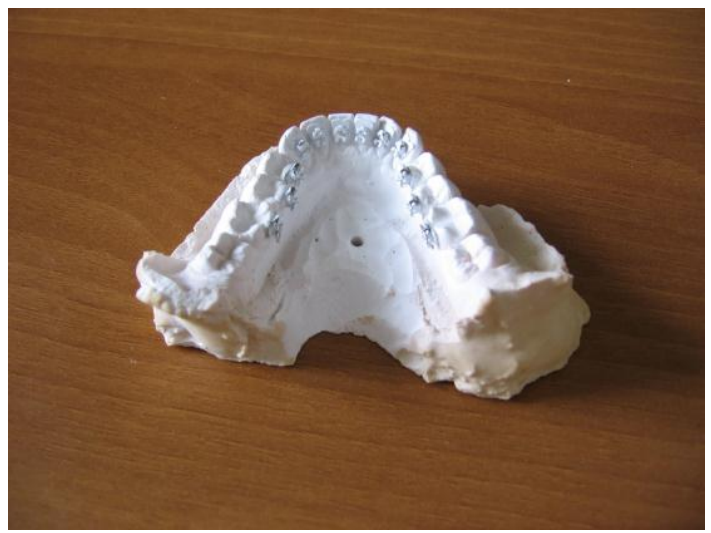

Figure 1. Plaster teeth arch copy with attached brackets

The disadvantages of traditional technique are

- The problems with installing standard wire in bracket's slot because of complex teeth position and orientation.

- Weak control on forces produced by standard wire sometimes resulting in tooth over shifting.

The new image-based technique is developed for overcoming these problems. It improves the existing one by introducing photogrammetric techniques for accurate $3 \mathrm{D}$ model producing and designing individual wire.

The proposed technique includes the next stages:

- Producing plaster copy of a teeth arch

- Positioning standard brackets on teeth using special device (articulator) which provides required bracket position on a tooth for producing correct forces for required tooth movement

- Producing accurate 3D model of teeth arch plaster copy

- Segmenting obtained 3D model into separate teeth and gum 3D models

- Positioning teeth 3D models in a required (correct) position according orthodontics' requirements

- Determining slot position for every bracket using image-based technique for spatial slot position

- Generating CAD model of an individual wire which accurately goes through the brackets slots

- Making a silicon kappa containing installed brackets in required position using 3D model of teeth arch with brackets

- Placing the brackets on a patient's teeth using this kappa which provide accurate brackets transfer to a given position for every tooth

- Placing an individual wire into the brackets

- Image-based monitoring of treatment process

Below the detailed description of proposed technique is given. 


\section{3D RECONSTRUCTION}

A photogrammetric system for automated teeth arch 3D model generation (Fig. 2.) includes two digital high resolution cameras, high resolution structured light projector and PCcontrolled rotation positioning stage. It allows producing high resolution 3D mjdels of teeth arch in automated mode.

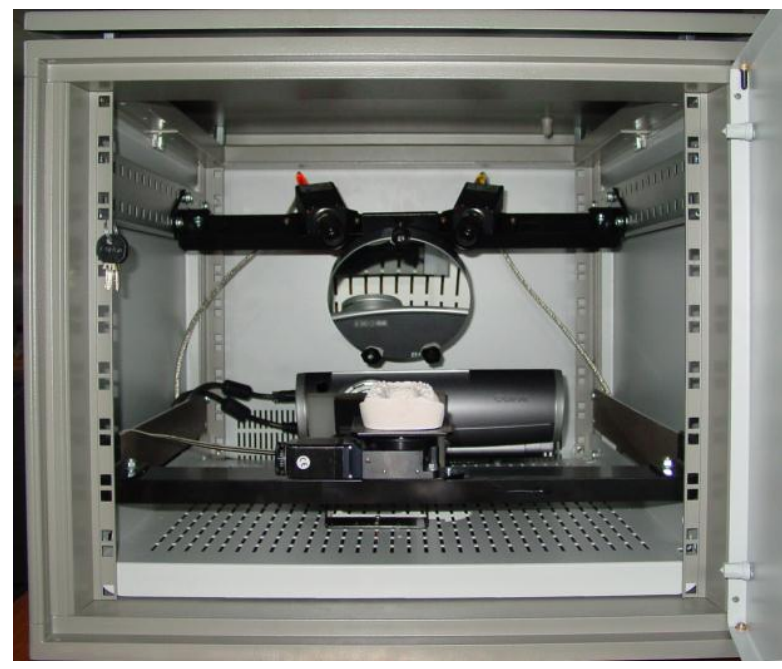

Figure 2. Photogrammetric system for teeth arch 3D model reconstruction

\subsection{Teeth 3D model generation}

The requirements to $3 \mathrm{D}$ reconstruction system were formulated according to investigation purposes:

- High accuracy of 3D reconstruction

- High resolution of tooth 3D model

- Short time for producing teeth arch 3D models

- The possibility of real occlusion registration and reconstruction

- The possibility of occlusion investigation

To meet these requirements the configuration of photogrammetric system including two digital IEEE 1394 cameras of high resolution (2 Mpixel), structured light projector with resolution of 1440x1080 pixels and PC-controlled rotation positioning stage is chosen. For automated corresponding problem solution coded light is applied providing robust and fast scanning.

The system is calibrated using the original technique for estimating camera interior and exterior orientation parameters and for determining the parameters of positioning stage rotation axis (Knyaz, V., 2005). Special calibration test field with coded targets is used for system parameters determination in automated mode. The calibration technique provides residuals of co-linearity conditions for the reference points after least mean square estimation at the level of $0.005 \mathrm{~mm}$. This accuracy is quite adequate for the concerning problem.

For whole teeth arch 3D model generation a set of scans at various plaster model positions is acquired. The number of scans and their orientation are the subject of user choice. All scans are transformed to global coordinate system using the results of rotation axis parameters estimation (Fig. 3.). This first order scans alignment serves as an initial approximation for accurate scan registration by iterative closest point algorithm (Besl, 1992).

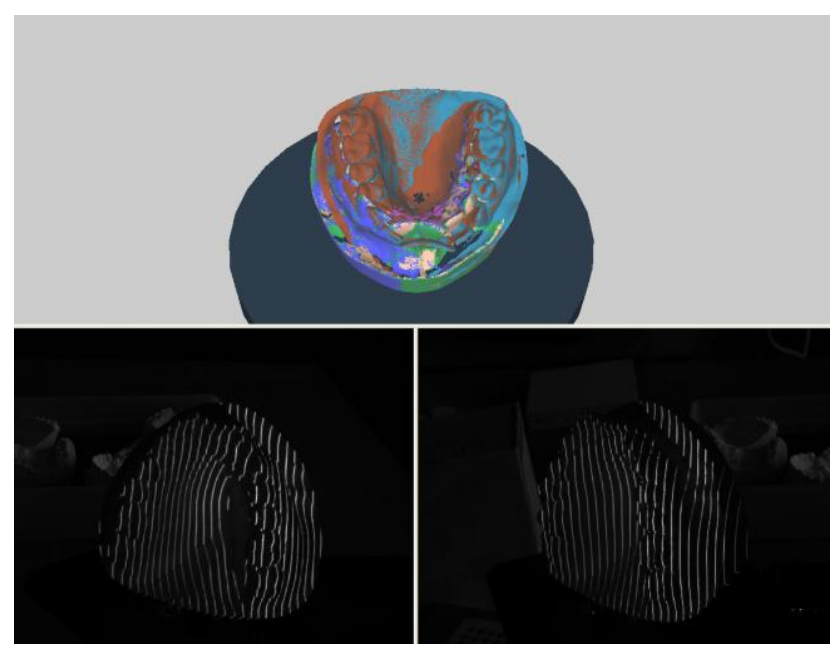

Figure 3. 3D reconstruction process

After scan merging a single mesh 3D model is generated using interpolating mesh algorithm.

For acquiring teeth arch 3D model using the described automated procedure it is required about 5 minutes. The resulting 3D model has accuracy of $0.03 \mathrm{~mm}$ and consist of about 1000000 points. Both upper and lower teeth arch 3D models are produced for further occlusion analysis.

\subsection{Teeth occlusion registration}

For occlusion registration special mode is used (Knyaz V., 2007). In occlusion registration mode both upper and lower teeth arch 3D model are placed in working space of scanning system and a 3D scan of teeth arches in occlusion is captured. Then this 3D scan is used for placing 3D models of upper and lower teeth arches in corresponding position.

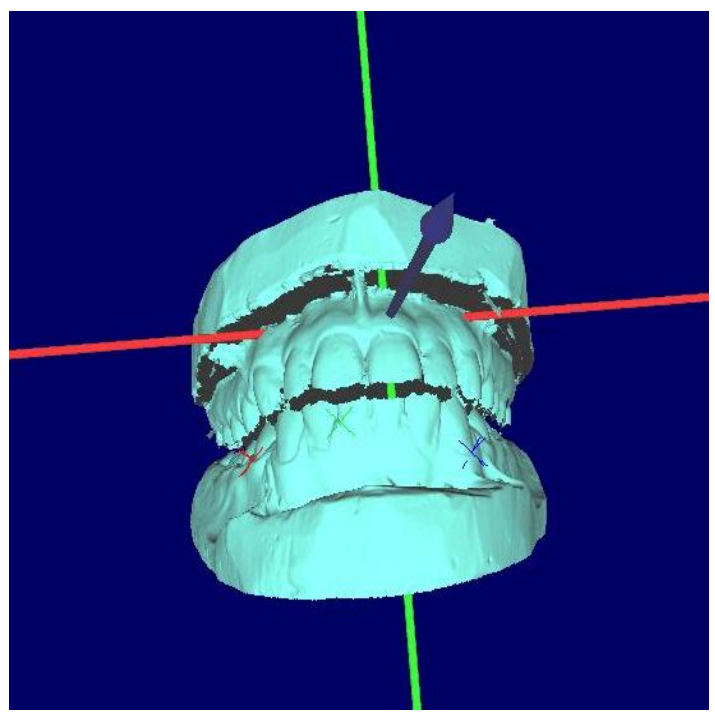

Figure 4. The scan of the teeth front surface for teeth arch 3D models positioning 
The first step is to install 3D models of upper and lower teeth arches in a given position according their location and orientation in a mouth. For this purpose plaster models are installed in given posture using a dental articulator or silicon mould for setup (Fig. 4). Dental articulator is used for registering central (normal) occlusion. Silicon mould is made by dentist in a mouth, jaws being in given position according left or right side occlusion.

The first developed technique for occlusion registration used a set of reference point on teeth observing by stereo system and virtual teeth arches were installed in occlusion position according to calculated 3D coordinates of this set of reference points. The new technique based on front teeth surface registration gives better accuracy and is chosen as general method for occlusion registration.

After the virtual setup procedure a dentist has teeth arches 3D models installed in a position according given occlusion, so he can perform all necessary investigations of the occlusion which he needs. A dentist can observe and study relations of opposite occlusion surfaces in different positions.

\section{VIRTUAL TEETH SET UP}

\subsection{D model segmentation}

For planning orthodontic treatment and individual wire design teeth arch 3D model is segmented into separate teeth and gum 3D models (Fig. 5).

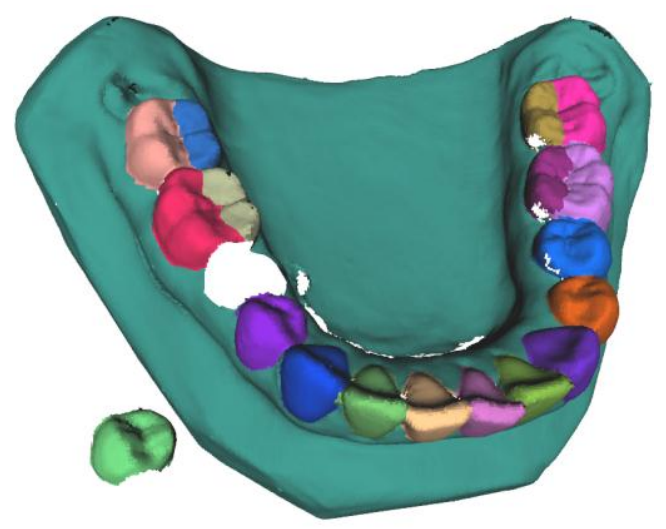

Figure 5. Results of teeth arch 3D model segmentation

The segmentation process is performed in semi-automatic mode using combined curvature-watershed algorithm. After segmentation teeth $3 \mathrm{D}$ models are transformed to watertight form. This form is needed for solving the collision detection problem during teeth shifting planning to avoid producing wrong-directed forces.

\subsection{Positioning teeth 3D models in a required (correct) position}

Segmentation allows calculating a set of tooth dimensions required for determining correct teeth position. Usually for calculating the perfect teeth arch curve mesio-distal dimensions of several teeth are used.

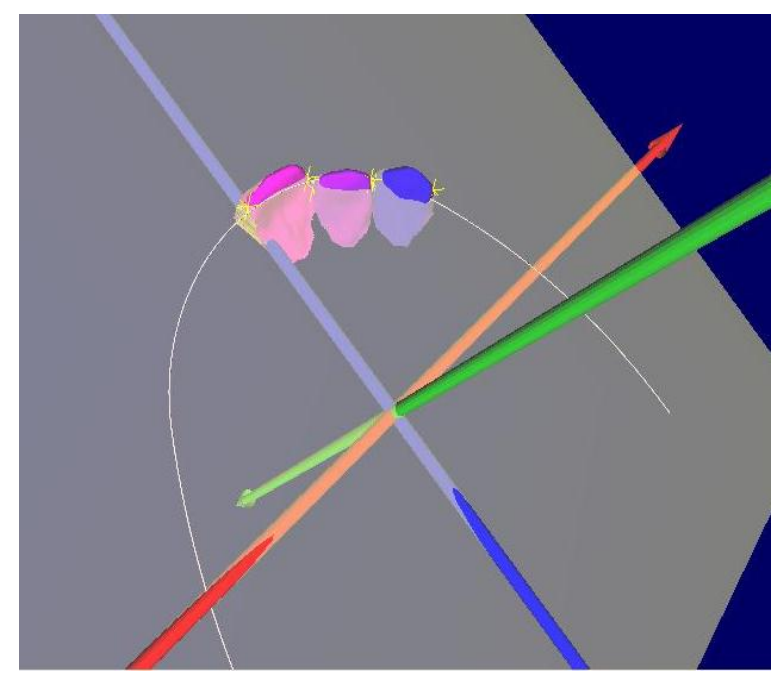

Figure 6. Perfect teeth arch shape calculation

Different method for determining perfect teeth arch curve could be applied in developed system. Figure 6. demonstrates the result of Hawley-Herber-Herbst curve determination based mesio-distal dimensions of three frontal teeth.

After perfect teeth arch shape determination virtual perfect teeth arch is generated so as all teeth would be in a correct position. Now brackets slots correspond to individual wire which is to move teeth into perfect position.

\section{INDIVIDUAL WIRE DISIGN}

\subsection{Determining slot position for every bracket}

The next task is to find spatial position of every slot and to design individual wire. Image-based technique for this problem is developed. It uses special slot probes with coded targets which allow finding with high accuracy the position and orientation of every slot in given coordinate system.

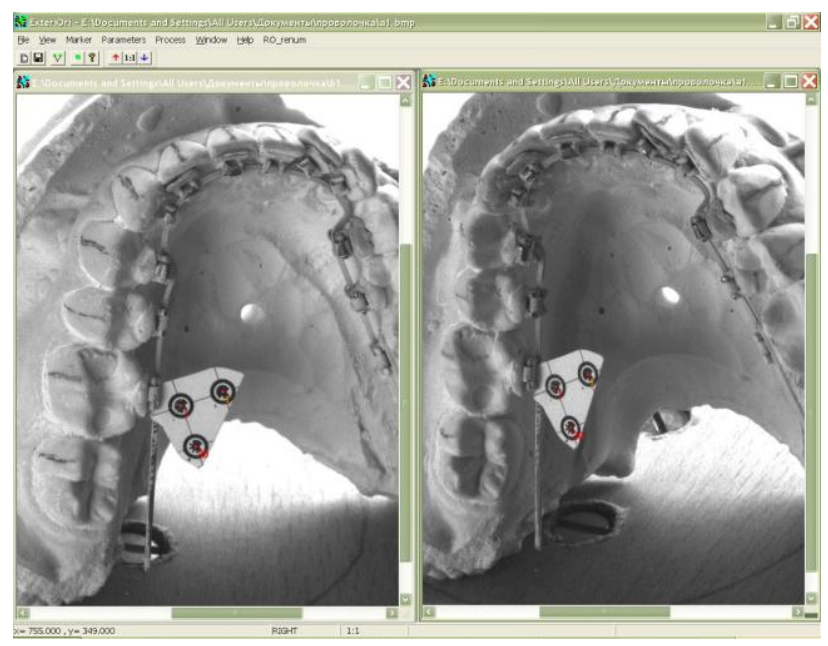

Figure 7. Image-based slot position determination

Slot position is estimated in automated mode. 
5.2 Generating CAD model of an individual wire which accurately goes through the brackets slots

The procedure of slot position determination results in slot 3D markers (Fig. 8), which are located in space accordingly slot position and orientation. These slot 3D markers then exported to required $\mathrm{CAD} / \mathrm{CAM}$ format for producing individual wire by chosen CAM technology.

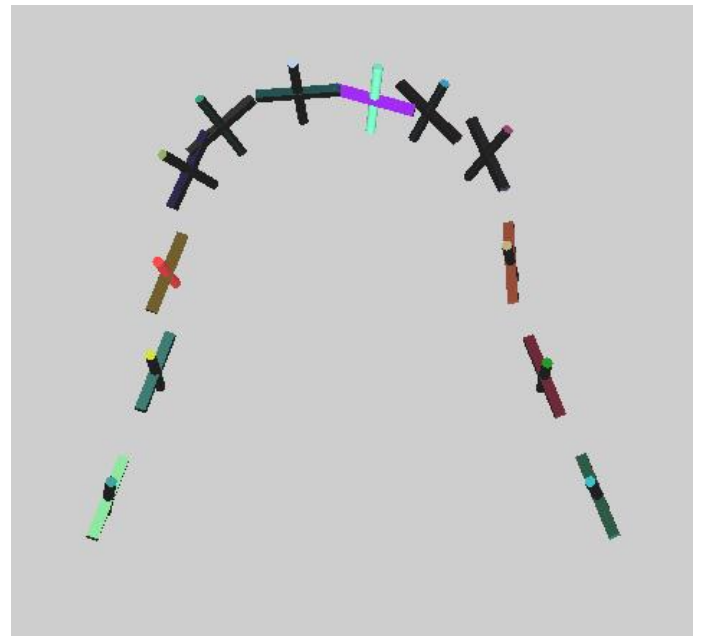

Figure 8. Slot 3D markers

The result of export to .dxf format for laser cutting machine is shown in Figure 9.

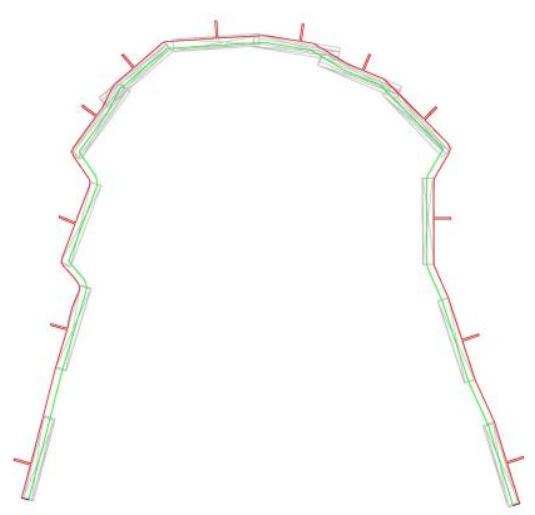

Figure 9. Slot positions export to .dxf format for automated wire production

\section{IMAGE-BASED MONITORING OF TREATMENT PROCESS}

For monitoring the process of teeth moving image-based technique is proposed. It uses the photograph of patient teeth arch at different stages of treatment and 3D model of teeth arch for current case. For analysing the results of treatment expert could use 3D model of teeth arch in initial or in perfect position.

Exterior orientation of the photograph based on a set of reference points is performed thus giving to the expert the possibility to analyse real teeth displacement relatively initial or desired condition.

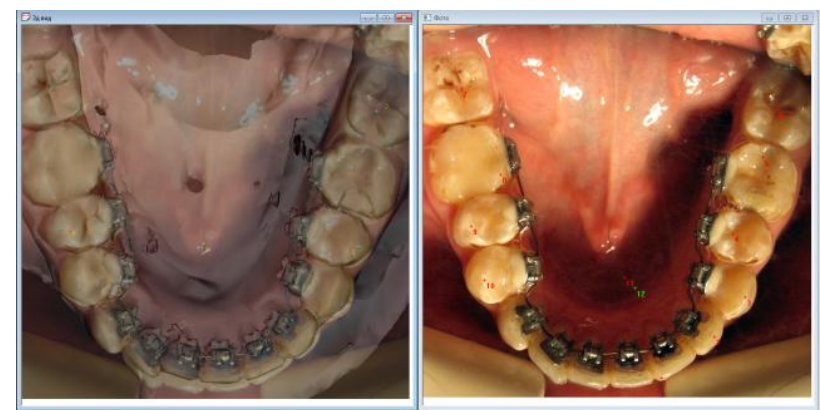

Figure 10. Image-based monitoring of treatment

\section{CONCLUSION}

The image based technique for orthodontic planning, treatment and documenting is proposed. It includes photogrammetric system for teeth arches 3D reconstruction, occlusion registration, accurate bracket's slot position determination.

The developed technique provides correct teeth arch set up based on occlusal plane determination and accurate teeth dimensions measurement. Virtual teeth setup is used for individual wire (a set of wires for staged treatment) design and automated manufacturing. An orthodontist has a mean for monitoring treatment process by comparing 3D model of teeth arch (in initial or desired condition) with oriented current photograph of patient teeth arch.

The developed technique was tested in orthodontic practice and demonstrated better results than traditional standard bracket technology.

\section{REFERENCES}

Knyaz V.A., Zheltov S.Yu., On-line calibration technique for mobile robot. EOS Conference on Industrial Imaging and Machine Vision, Munich, Germany, June 13-15, 2005, pp. 9597

Besl, P.J., McKay, N.D., 1992: A method for registration of 3D shapes, IEEE Transactions on Pattern Analysis and Machine Intelligence, vol. 14, no.2, pp. 239-256

Knyaz V., Zheltov S.., Gaboutchan A., Bolshakov G. Photogrammetric system for automated teeth arches 3D models generation and teeth occlusion analysis. Proceedings of "Optical 3D measurements techniques" 2007, Zurich, Switzerland, pp. 135-140 